\title{
Accelerated long-term forgetting in resected and seizure-free temporal lobe epilepsy patients
}

${ }^{1}$ Visser, M., ${ }^{1}$ Forn, C., ${ }^{2}$ Gómez-Ibáñez, A., ${ }^{1}$ Rosell-Negre, P., ${ }^{2}$ Villanueva, V., ${ }^{1}$ Ávila, C.

${ }^{1}$ NNF, departament de Psicología Bàsica, Clínica i Psicobiología, Universitat Jaume I, Castellón, Spain.

${ }^{2}$ Multidisciplinary Epilepsy Unit, Hospital Universitario y Politécnico La Fe, Valencia.

Figures: 3

Tables: 4

Pages: 28

Words Abstract: 226

Corresponding author: Maya Visser, department of PsicologíaBásica, Clínica y Psicobiología ,Universitat Jaume I, Avda. SosBaynat s/n, 12071, maya.visser@gmail.com, phone number: +349582246789, fax number: +34964729267

Acknowledgements: This research was supported by the Spanish Ministry of Science and Innovation (PSI2013-45378-R) and the University Jaume I, Castellon (P1·1B2014-03).

Conflicts of interest: none 


\begin{abstract}
Episodic memory impairments caused by temporal lobe epilepsy (TLE) are well documented in the literature. Standard clinical episodic memory tests typically include a 30-minute delayed recall test. However, in the past decade, it has become apparent that this standard test does not capture the full range of memory problems in TLE patients. Some patients perform well on a standard 30-minute delayed recall test, but show Accelerated Long-term Forgetting (ALF) after 24 hours. Although ALF has been investigated in patients with different types of epilepsy, current research on resected TLE patients is missing. In the present study, resected TLE patients were compared to a control group matched on initial learning. They showed normal performance on verbal recall after 30 minutes, but impairments became apparent after one week. Moreover, the significant interaction between participant group and memory test delay demonstrated that the patients indeed showed an acceleration in forgetting. Furthermore, ALF was present in both left and right resected TLE patients, which contradicts the presence of material-specific hemispheric differences in ALF. In addition, ALF was observed in seizure-free resected TLE patients, thereby demonstrating that this factor is not crucial for long-term memory deficits. The outcome shows that clinicians are likely to underestimate memory deficits in resected TLE patients and, therefore, advocates for the inclusion of ALF tests in standard clinical batteries for both pre- and post-surgery testing sessions.
\end{abstract}




\section{Introduction}

Episodic memory refers to autobiographical events that can be explicitly stated (i.e., awareness of the time, place, and other contextual circumstances). For example, people can remember when, where, and with whom they celebrated their $20^{\text {th }}$ birthday. A vast amount of literature has identified the hippocampus as an essential structure for episodic memory. Consequently, episodic memory problems are well documented in mesial TLE patients with hippocampal sclerosis and temporal lobe resection (for reviews see: Bell, Lin, Seidenberg, \& Hermann, 2011; Moscovitch et al., 2016). Based on traditional models, the fixation of information in the long-term memory is thought to take place within 30 minutes (Hoefeijzers, Dewar, Della Sala, Butler, \& Zeman, 2015; Jansari, Davis, McGibbon, Firminger, \& Kapur, 2010; Wilkinson et al., 2012). As a result, clinicians and researcher have developed standard 30-minute delayed recall tests, which have been successful in defining episodic memory impairments (Kilpatrick et al., 1997; Lencz et al., 1992; Pauli, Hildebrandt, Romstock, Stefan, \& Blumcke, 2006; Rausch \& Babb, 1993; Reminger et al., 2004). However, there is a common mismatch between subjective complaints and objective memory performance: some TLE patients can perform well on a standard episodic memory test, but still complain about memory problems (Butler \& Zeman, 2008; Piazzini, Canevini, Maggiori, \& Canger, 2001), which indicates that these standard tests might not always capture the full range of memory problems in TLE patients (Butler \& Zeman, 2008).

\subsection{Accelerated long-term forgetting in TLE patients}

In the past decade, a number of individual case and group studies have reported nonresected TLE patients who pass the standard clinical memory tests, but complain about long-term memory problems (Blake et al., 2000; Helmstaedter, Hauff, \& Elger, 1998; Jansari et al., 2010; Narinder Kapur et al., 1997; Kemp et al., 2012; Lucchelli \& Spinnler, 1998; Mameniskiene et al., 2006; Martin et al., 1991; Mayes et al., 2003; McGibbon \& Jansari, 2013; O’Connor et al., 1997; Ricci et al., 2015; Wilkinson et al., 2012). These studies have typically included the standard 30-minute delay, with additional testing at longer intervals ranging between one day and eight weeks. Although the patients performed normally on the 30-minute interval, they showed impaired performance on the longer intervals. This phenomenon is called "long-term 
amnesia" (LTA; Kapur et al., 1997), or more recently, "accelerated long-term forgetting” (ALF; Butler \& Zeman, 2008; Blake et al. 2000).

To investigate ALF, patients are normally presented with the same material repeated over various trials during the learning phase. After this, they are required to recall as much as possible during the various recall phases. Whereas some studies have used a verbal word list as material (e.g., Bell, Fine, Dow, Seidenberg, \& Hermann, 2005; Martin et al., 1991), others have assessed the patients with a story (e.g., Bell, 2006; Blake, Wroe, Breen, \& McCarthy, 2000; Wilkinson et al., 2012). In addition, some researchers have tested for non-verbal episodic memory by letting patients copy easy drawings (Giovagnoli, Casazza, \& Avanzini, 1995) or by including the ReyOsterrieth Complex Figure Recall test (Mameniskiene et al., 2006; Wilkinson et al., 2012).

Several studies found impaired performance after 30 minutes as well as after longer delays, but without a group by delay interaction to indicate ALF (Bell, 2006; Bell et al., 2005; Giovagnoli et al., 1995). Therefore, they concluded that ALF over 24 hours is uncommon in TLE patients. However, Wilkinson et al. (2012) pointed out that the lack of interaction might be due to the fact that the patients and controls were not matched on the short delays. In 1978, Huppert and Piercy suggested matching patients' initial levels of learning with that of their control group to avoid scaling problems due to their differences in performance (in Isaac \& Mayes, 1999). Specifically, when groups are mismatched on initial learning, forgetting rates can be underestimated in the lowerperforming group as they have less to forget.

Indeed, various studies that matched TLE patients and controls at early levels of learning found evidence of ALF in TLE patients (Blake et al., 2000; Cassel et al., 2016; Martin et al., 1991; Wilkinson et al., 2012). For example, Martin et al. (1991) and Blake et al. (2000) found normal performance at the 30-minute delay, but impaired performance after 24 hours and eight weeks, respectively. In addition, their results demonstrated a group by delay interaction, suggesting ALF. By contrast, in the study of Wilkinson et al. (2012), TLE patients showed impaired recognition after both 1 hour and 6 weeks. However, unlike in the studies of Bell and Giovagnoli (Bell, 2006; Bell et al., 2005; Giovagnoli et al., 1995; see previous paragraph), they did find a group by delay interaction. Wilkinson et al. (2012) thereby opened up the debate about the definition of ALF. The original description of ALF stresses the presence of intact 
performance after delays of up to 1 hour, accompanied by impairments after longer delays (Butler et al., 2008). However, Wilkinson et al. (2012) state that the relative rates of forgetting are important, as measured by the interaction between the participant group and the memory test delay. Accordingly, Wilkinson et al. (2012) concluded that their TLE patients demonstrated ALF.

\subsection{The neural substrates underlying $A L F$}

The phenomenon ALF poses a challenge to standard clinical measures as well as to the underlying theoretical assumptions (Butler \& Zeman, 2008; Jansari, Davis, McGibbon, Firminger, \& Kapur, 2010). The influential model on memory consolidation by Alvarez and Squire (1984) includes two stages: the information that binds together to form a memory component is initially stored in the medial temporal lobe (MTL) and then shifts laterally to the neocortex (in Squire \& Alvarez, 1995). Although this process may continue for longer periods, it is often assumed that the greater part of consolidation is completed in a relatively short time frame and can be effectively tested within 30 minutes (Butler \& Zeman, 2008; Jansari et al., 2010; Wilkinson et al., 2012). Based on this model, Mayes et al. (2003) suggested that ALF is apparent in patients with neocortical damage (e.g., anterior temporal lobe and orbitofrontal cortex), whereas medial damage is rare. They speculated that the intact MTL in TLE patients with ALF allows initial consolidation of binding information, whereas transfer of this information to, or its maintenance within the long-term storage sites in the neocortex, may be impaired. However, more recent studies have shown that structural brain damage in medial temporal regions dominates the clinical profiles associated with ALF (Zeman, Butler, Muhlert, \& Milton, 2013). A number of studies have demonstrated that ALF can be related to increased T2 relaxation times in the hippocampus (Wilkinson et al., 2012), reduced hippocampal volume (Butler et al., 2009; Muhlert, Milton, Butler, Kapur, \& Zeman, 2010), and hypometabolism in the MTL (Tramoni et al., 2011).

Assuming that the involvement of the MTL is restricted to the first stage of memory consolidation (as suggested by Alvarez and Squire in 1984), the continuing consolidation process after 30 minutes would be more important than originally thought and essential to ensuring proper preservation of information. Alternatively, it could mean that episodic memories will always remain dependent on the MTL, through 
ongoing rehearsal and reactivation during the stabilisation and retrieval processes, as suggested by the Multiple Trace Theory of Nadel and Moscovitch (1997).

\subsection{ALF caused by epileptiform activity}

Structural damage to the hippocampus might not be the only factor that leads to ALF in TLE patients (Wilkinson et al., 2012; Zeman et al., 2013). For example, TLE patients in the study of Blake et al. (2000) demonstrated ALF despite an intact hippocampus, as indicated by Magnetic Resonance Imaging (MRI). ALF has been related to overt clinical seizures (Mameniskiene et al., 2006; O’Connor et al., 1997; Wilkinson et al., 2012). However, another study found no association between seizures and ALF (Muhlert et al., 2010). In these cases, subclinical epileptiform activity could be a cause. In agreement, the study of Mameniskiene et al. (2006) indeed showed that interictal discharges on the EEG correlate with ALF impairments. Furthermore, ALF has been reported in other disorders involving seizure activity, such as transient epileptic amnesia (TEA; Butler et al., 2009; 2008; 2007; 2008). These patients experience brief episodes of isolated amnesia as a result of seizure activity in the medial temporal lobes (Butler et al., 2007; Kapur, 1990; Zeman \& Butler, 2010). ALF in these patients seems to be unrelated to the frequency of overt seizures (Butler et al., 2009). Moreover, TEA patients with ALF are typically seizure free with medication use (Butler et al., 2007; Muhlert et al., 2010). It was recently proposed that subclinical epileptiform activity during sleep might lead to ALF in these patients (Muhlert et al., 2010; Zeman et al., 2013). Overall, Zeman et al. (2013) emphasize that the underlying origin of the disorder can be attributed to structural anomalies or epileptiform activity, or a combination of the two. For example, ALF can be observed in patients with epileptiform activity, but without structural brain damage, and vice versa (Zeman et al. 2013).

\subsection{ALF in left vs. right TLE patients}

Many studies have suggested the presence of a material-specific difference in memory functions of the left and right temporal lobes (e.g., Milner, 1971). An extensive review of the neuroimaging data observed that verbal and non-verbal episodic memory rely dominantly on the left and right temporal lobes, respectively (Cabeza \& Nyberg, 2000). Congruently, the laterality of the seizure focus has consistently been found to influence 
the type of material for which memory is most affected, with left TLE causing more pronounced deficits in verbal memory (Baxendale et al., 1998; Delaney, Rosen, Mattson, \& Novelly, 1980; Hermann, Seidenberg, Schoenfeld, \& Davies, 1997; Mungas, Ehlers, Walton, \& McCutchen, 1985). Furthermore, research has associated right TLE with visuo-spatial memory impairments, although this relationship is less consistent (Barr, Goldberg, Wasserstein, \& Novelly, 1990; Baxendale et al., 1998; Gleissner, Helmstaedter, \& Elger, 1998; Lee, Yip, \& Jones-Gotman, 2002). Alternatively, it has been suggested that visuo-spatial memories may rely on a dynamic bilateral interaction between MTL structures (Glikmann-Johnston et al., 2008). However, with regard to ALF, laterality effects are inconclusive (for reviews see: Butler \& Zeman, 2008; Elliott, Isaac, \& Muhlert, 2014). Some studies on ALF do not include laterality as a study factor (e.g., Mameniskiene et al., 2006; Martin et al., 1991). In addition, studies that separate left and right TLE patients show inconsistent results. Blake et al. (2000) found ALF for verbal story tests in left, but not right, TLE patients. In contrast, Wilkinson et al. (2012) found ALF effects for verbal story information in both left and right TLE patients. One potential explanation for the differences in verbal recall is that left and right TLE patients were categorised differently. Whereas Wilkinson et al. (2012) used hippocampal pathology to define lateralisation, Blake et al. (2000) focused on seizure activity. Wilkinson et al. (2012) pointed out that, even though hippocampal damage was lateralised in their patients, seizure activity might not be. In other words, right TLE patients might still cope with left MTL seizures, causing verbal memory problems. In addition, they pointed out that the hippocampal pathology of the patients in the study of Blake et al. (2000) was of a lesser degree. Another potential factor that can explain the differences between the study of Blake et al. (2000) and Wilkinson et al. (2012) is their use of a different procedure. Whereas the material in the former study was repeated until reaching a performance level of over $90 \%$ correct, the latter study required only 75\% accuracy. Bell (2006) argued that using high levels on initial performance poses the risk of material being over-learnt, leading to the possibility that early forgetting is masked by ceiling effects. For a more detailed discussion on this issue, see the review of Elliot et al. (2014). Finally, Wilkinson et al. (2012) found that right, but not left, TLE patients showed ALF on the Rey-Osterrieth Complex Figure Recall test.

Overall, the pattern of material-specific laterality effects in ALF is unclear, which is largely due to a lack of research on this issue. However, based on the 
aforementioned literature, it is important to separately investigate the performance of left and right TLE patients.

\subsection{Investigating ALF in resected TLE patients}

Overall, based on traditional models, the fixation of information in the long-term memory is thought to largely take place within 30 minutes. However, in recent decades, it has been suggested that subsequent consolidation processes are required to ensure proper preservation of the information. These observations reveal the need for broader episodic memory batteries to capture the full range of memory problems in TLE patients.

The current study will investigate ALF in resected TLE patients. All patients underwent standard en bloc resection. This means that hippocampal pathology was homogeneous across the patient groups. Hippocampal volume in TLE patients has been associated with memory performance, introducing possible confounding variations in studies on non-resected patients (Wilkinson et al., 2012). As the patients in the current study had standard hippocampal resection, the possible influencing factor of hippocampal volume variations is removed. Furthermore, inconsistent results on material specific laterality effects of ALF were attributed to differences in hippocampal pathology (Wilkinson et al., 2012). Therefore, the current study can shed light on these laterality effects. To our knowledge, the current study is the first to investigate ALF in resected TLE patients (with the exception of two studies that included mixed groups of resected and non-resected TLE patients; Martin et al., 1991; Ricci, Mohamed, Savage, \& Miller, 2015).

\section{Methods}

\subsection{Participants}

Sixty participants were included in the current study, 30 resected TLE patients and 30 control participants. All participants were tested by an experienced neuropsychologist, and the study was approved by the ethical committees of Jaume I University, Castellón, and la Fé Hospital, Valencia, Spain. 


\subsubsection{Patient group}

Thirty adult patients $(\mathrm{N}=15$ male; $\mathrm{N}=28$ right -handed) were recruited retrospectively from the Multidisciplinary Epilepsy Unit at La Fe Hospital (Valencia, Spain). All patients underwent standard antero-temporal lobectomy with amygdalohippocampectomy $(\mathrm{ATL}+\mathrm{AH}), 13$ in the left hemisphere and 17 in the right one, after being diagnosed with mesial TLE due to hippocampal sclerosis. All of them had been considered good surgical candidates after a pre-surgical evaluation that included prolonged video-EEG monitoring, a 3-Tesla brain MRI, a psychiatric evaluation, and neuropsychological testing. None of the patients had a psychiatric disorder. The standard resection included removal of the head and body of the hippocampus while preserving the tail. The lateral extent of the temporal lobe resections was $4.5 \mathrm{~cm}$ from the temporal tip in the dominant hemisphere and $5.5 \mathrm{~cm}$ in the non-dominant hemisphere. Furthermore, the superior temporal gyrus was preserved. Mean age at epilepsy onset was 8.9 years (range 1-40), and surgery had been performed 27.8 years (range 1-59) after onset. At the time of our study, the mean age of the patients was 41.3 years (range 19-62). Twenty-six patients were in the late post-operative phase (after 12 months), and four patients were in the early post-operative phase (between 6 and 12 months) (months post-surgery $M=55.9 \pm 30.3$ ). Seizure frequency decreased in all patients after surgery. Sixteen patients were seizure-free (Engel class I), four had rare disabling seizures (Engel class II), and ten had experienced worthwhile improvements (Engel class III) since the surgery. Individual patient data regarding epilepsy features are summarized in Tables 1 and 2.

\subsubsection{Age, education, and IQ matched control group}

Patients were compared to an education- and age-matched control group of 30 participants $(\mathrm{n}=14$ male, $\mathrm{n}=24$ right-handed) without neurological or psychiatric impairments. As described in Method section 2.3, the control group was matched with the patient group on the fifth trial of immediate learning, which meant that we excluded 12 control participants from the analyses. For the remaining 18 control participants, we ensured that they matched the left TLE and right TLE patients on age, handedness and gender $\left(\right.$ age: $F(2,46)=2,15, p=0.13$; handedness $X^{2}(2)=0.369, p=0.83$; gender $X^{2}$ $(2)=.49, p=.78)$. Furthermore, we checked the years of education for the three groups, 
which differed significantly $(F(2,46)=4,02, p=0.045)$ because the control group had studied fewer years: left TLE: $\mathrm{M}=11.38 \pm 3.0$; right TLE: $\mathrm{M}=11.23 \pm 3.3$; controls: $\mathrm{M}=9.22 \pm 2.9$. However, this is not a confounding factor because impaired memory in patients is unlikely to be related to more years of education. Furthermore, we included the Matrix reasoning test of non-verbal intelligence (a subtest of the Wechsler adult intelligence Scale III: WAIS III; Kaufman \& Lichtenberger, 2006) to obtain the intellectual quotient (IQ) value based on non-verbal items. An F-test of left TLE, right TLE, and controls confirmed that their matrix reasoning did not differ $(F(2,46)=0.372$, $\mathrm{p}=0.70$; left TLE: $\mathrm{M}=99.00 \pm 17$; right TLE: $\mathrm{M}=101.77 \pm 15$; controls: $\mathrm{M}=102.22 \pm$ 8). In short, we carefully chose participants matched on education, age, and matrix reasoning to avoid group differences to be caused by merely a difference in intelligence (according to this scale) or education. We are thereby eliminating an important bias that is commonly present in patient studies (Butler \& Zeman, 2008; Elliott et al., 2014; Miller, Flanagan, Mothakunnel, Mohamed, \& Thayer, 2015).

\begin{tabular}{lllllll}
\hline Patient & Gender & Age & $\begin{array}{l}\text { Age at } \\
\text { onset }\end{array}$ & $\begin{array}{l}\text { Age at } \\
\text { surgery }\end{array}$ & $\begin{array}{l}\text { Surgery } \\
\text { outcome* }\end{array}$ & Treatment** \\
\hline 1 & M & 37 & 1 & 28 & I & OXC, CZP \\
2 & M & 59 & 14 & 58 & I & LTG, VPA, LCM \\
3 & M & 62 & 5 & 58 & I & LCM, CBZ \\
4 & F & 47 & 1 & 46 & I & CZP, CBZ \\
5 & M & 39 & 3 & 32 & I & CBZ \\
6 & F & 39 & 19 & 33 & II & LEV, OXC, VPA \\
7 & F & 29 & 1 & 26 & I & LEV, LCM, CBZ \\
8 & M & 44 & 4 & 36 & I & CBZ, LEV \\
9 & M & 54 & 14 & 49 & II & LTG \\
10 & M & 50 & 18 & 42 & III & CBZ, CLB \\
11 & F & 33 & 27 & 32 & III & CBZ \\
12 & M & 50 & 18 & 49 & I & LCM, VPA, LEV \\
13 & F & 47 & 2 & 38 & II & PB, LCM, LTG, CLB \\
14 & F & 43 & 29 & 35 & III & CBZ, LCM, BRV \\
15 & M & 47 & 12 & 46 & I & OXC, LCM \\
16 & F & 33 & 5 & 30 & II & ESL, LCM, PER \\
17 & F & 50 & 1 & 45 & III & TPM, CLB \\
\hline
\end{tabular}

Table 1. Demographics of the right TLE patients. M: male; F: female. Age in years. Engel classification*: I: free of disabling seizures; II: rare disabling seizures; III: worthwhile improvement in seizures; IV: no worthwhile improvement in seizures. Treatment**: OXC: 
oxcarbazepine; CZP: clonazepam; LTG: lamotrigine; VPA: valproic acid; LCM: lacosamide; CBZ: carbamazepine; LEV: levetiracetam; CLB: clobazam; PB: phenobarbital; BRV: brivaracetam; PER: perampanel; PHT: phenytoin; TPM: topiramate.

\begin{tabular}{|c|c|c|c|c|c|c|}
\hline Patient & Gender & Age & $\begin{array}{l}\text { Age at } \\
\text { onset }\end{array}$ & $\begin{array}{l}\text { Age at } \\
\text { surgery }\end{array}$ & $\begin{array}{l}\text { Surgery } \\
\text { outcome* }\end{array}$ & Treatment** \\
\hline 1 & $\mathrm{M}$ & 19 & 5 & 16 & III & LTG, LCM, PER \\
\hline 2 & $\mathrm{M}$ & 33 & 1 & 30 & I & LEV, LTG \\
\hline 3 & $\mathrm{~F}$ & 22 & 15 & 16 & I & CBZ, CLB \\
\hline 4 & $\mathrm{M}$ & 34 & 1 & 27 & I & VPA, LTG \\
\hline 5 & $\mathrm{~F}$ & 24 & 1 & 19 & I & LTG \\
\hline 6 & M & 57 & 14 & 51 & I & LCM, LTG, CLB \\
\hline 7 & $\mathrm{~F}$ & 49 & 1 & 45 & III & CBZ, LEV, LCM \\
\hline 8 & $\mathrm{~F}$ & 34 & 19 & 29 & III & CBZ, CLB \\
\hline 9 & $\mathrm{M}$ & 49 & 40 & 43 & III & VPA, LEV, PHT \\
\hline 10 & $\mathrm{~F}$ & 48 & 10 & 40 & I & $\mathrm{CBZ}, \mathrm{CZP}$ \\
\hline 11 & $\mathrm{~F}$ & 29 & 11 & 29 & I & VPA, LCM \\
\hline 12 & $\mathrm{M}$ & 59 & 8 & 56 & III & LEV, LCM, CLB \\
\hline 13 & $\mathrm{~F}$ & 30 & 9 & 25 & III & CBZ, LCM, LEV, CLB \\
\hline
\end{tabular}

\subsection{Neuropsychological testing}

All participants were tested by an experienced neuropsychologist. After a preliminary interview, participants performed the Rey Auditory Verbal Learning Test (RAVLT; Rey, 1958), which is an established neuropsychological test to measure the ability to encode, store, and recover verbal information. During this test, they are presented with a list of 15 unrelated words repeated over five different trials, and each time they are asked to repeat as many as they can remember. In addition, two follow-up sessions are held a 30 minutes and after seven days. In these sessions, they are asked to recall the items without reading them again. After this, they are presented with a recognition list that includes the original 15 learned items and 15 foils. Rehearsal effects are prevented by performing the Matrix Reasoning Subtest of the Wechsler Adult Intelligence Scale 
(WAIS III; see section 2.1.1 of this manuscript) in the 30 minute delay of the RAVLT. In addition, participants were not informed about the second testing session, which was conducted over the phone. This ensures that participants were not aware of the future session and would not be motivated to rehearse (Elliott et al., 2014).

\subsection{Equating groups on immediate recall}

Several scientific articles on ALF have concluded that it is important to match groups on initial learning to avoid scaling problems due to differences in performance, including ceiling and floor level effects (Butler \& Zeman, 2008; Elliott et al., 2014; Isaac \& Mayes, 1999a; Wilkinson et al., 2012). Several methods have been suggested to equate initial learning, such as case-to-case matching, extended exposure times, and learning to criterion (for reviews see; Butler \& Zeman, 2008; Elliott et al., 2014). The majority of these methods require creating new tests or adapting standard episodic tests. However, in collaboration with the neurologists, we decided to use the original RAVLT set-up, thereby allowing its usage for further clinical and research purposes beyond the current study. We subsequently excluded control participants who recalled over $90 \%$ on the last learning trial. A Kruskal-Wallis test showed that the remaining control group was matched on initial learning (i.e., on the fifth learning trial) with the TLE patient groups $(\mathrm{H}(2)=3.01, \mathrm{p}=0.22)$. This additionally ensures that the control data is not confounded by ceiling effects. The disadvantage of this method is that these 'lower range' control participants might not properly represent the population (Butler et al., 2008). More specifically, the exclusion of cognitively normal controls from the comparison decreases the representativeness of this population and may lead to systematic "unmatching" (Meehl, 1970). However, the advantage is that we were able to use the standardized RAVLT without any adaptations (except the addition of a 7-day recall phase). Using this format will make it easier for clinicians to implement ALF testing in their standard memory batteries without increasing the examination time.

\subsection{Statistical analyses}

We will first analyse all the patients as one combined group, followed by analyses of the left and right TLE subgroups separately, keeping to the template provided by Blake et al. (2000) and Wilkinson et al. (2012). In addition, we will present the results for 
patients with and without seizures separately, allowing the comparison of the two patient profiles.

The encoding phase of the RAVLT included five repetitions of the word list, followed by 30 -minute and one-week delayed recall phases. We will look for impaired episodic memory after 30 minutes and after one week, as well as a group by delay interaction to investigate ALF.

Furthermore, Kolmogorov-Smirnov tests revealed that several of our analyses coped with a non-normal distribution. The control group showed a non-normal distribution on the fifth learning trial $(\mathrm{D}(18)=0.22, \mathrm{p}=0.025)$ and on the 30 -minute and one-week delay recognition task $(\mathrm{D}(18)=0.25, \mathrm{p}=0.004$ and $\mathrm{D}(18)=0.27, \mathrm{p}=0.001$, respectively). The left TLE group showed a non-normal distribution on the one-week delayed recall test $(\mathrm{D}(13)=0.27, \mathrm{p}=0.01)$. The right TLE group showed a non-normal distribution on the 30-minute delayed recognition task $(D(16)=0.22, p=0.04)$. This, in addition to a relatively small sample size, led us to decide to use non-parametric tests. We used the Mann-Whitney test to investigate group differences. Interaction analyses were conducted using a non-parametric ranking method described by Leys and Schumann (2010).

\section{Results}

\begin{tabular}{lllll}
\hline & Left TLE & Right TLE & $\begin{array}{l}\text { Matched } \\
\text { Controls }\end{array}$ & All Controls \\
\hline Learning trial 1 & $5.08(1.38)$ & $5.44(1.55)$ & $5.22(1.48)$ & $6.27(1.95)$ \\
Learning trial 2 & $7.38(1.45)$ & $7.75(1.91)$ & $8.11(1.75)$ & $9.33(2.14)$ \\
Learning trial 3 & $8.31(1.84)$ & $9.19(1.87)$ & $9.28(1.90)$ & $10.63(2.41)$ \\
Learning trial 4 & $9.38(1.85)$ & $9.94(2.26)$ & $10.67(1.94)$ & $11.7(2.09)$ \\
Learning trial 5 & $10.08(2.10)$ & $10.38(2.31)$ & $11.28(1.81)$ & $12.57(2.14)$ \\
30 min recall & $7.15(2.94)$ & $8.12(2.23)$ & $8.06(2.41)$ & \\
Week recall & $3.23(1.74)$ & $3.94(2.16)$ & $5.39(2.12)$ & \\
30 min recogn & $13.23(1.74)$ & $12.81(2.43)$ & $13.28(3.29)$ & \\
Week recognition & $10.08(3.45)$ & $11.13(3.14)$ & $11.01(3.88)$ & \\
\hline
\end{tabular}

Table 3. Means and standard deviations of the patient and control groups on the various sections of the RAVLT. 


\subsection{Verbal learning curve}

The first part of the RAVLT includes five learning trials (see Method section 2.2). The black line in Figure 4 represents the learning curve of the control group, including the original 30 controls. Kruskal-Wallis tests showed that this group performed better than the left and right TLE patients on levels 2 to 5 ( $\mathrm{p}<0.005)$. However, as explained in the Method Section, we matched controls and patients on immediate learning. To do this, we excluded control participants who scored over $90 \%$ correct on the fifth learning trial. The remaining control group included 18 participants, and their learning curve is represented by the dark-grey line in Figure 1. When comparing the patient groups and matched controls, the significant effects disappeared on all five levels $(p>0.2)$. This means that the performance on immediate learning was matched for the three groups and, therefore, would not influence the ALF analyses.

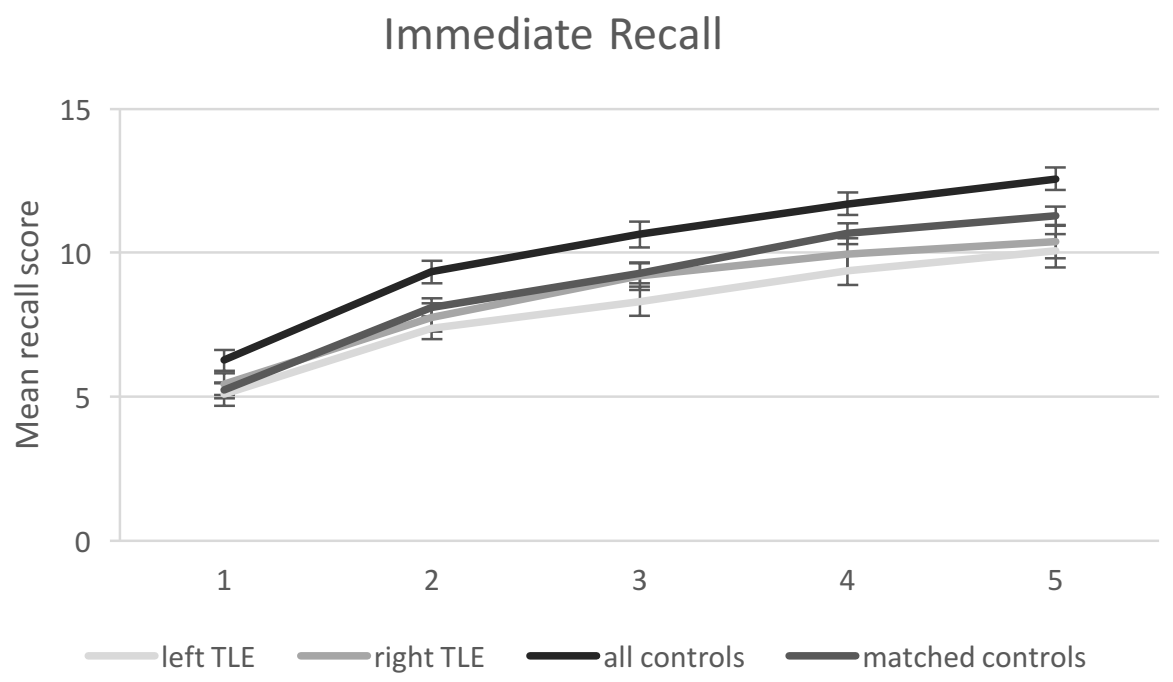

Figure 1 shows the learning curve of the left and right TLE patient groups (blue and red, respectively). In addition, the yellow line indicates the control group, including all 30 original controls. However, as explained in the method section, 12 controls were excluded from the analyses in order to match the groups on immediate recall. The learning curve of the remaining 18 controls is represented by the grey line. Error bars represent SEM. 


\subsection{ALF in TLE patients}

First, we tested performance decline on the 30-minute delayed recall. The MannWhitney test showed that verbal recall was not affected in TLE patients after 30 minutes $(\mathrm{U}=193.00, \mathrm{p}=0.10, \mathrm{r}=-0.04)$. In addition, we examined the loss between immediate recall on the fifth trial and the delayed recall after 30 minutes. The group $(2) * \operatorname{delay}(2)$ interaction was not significant $(\mathrm{F}(1,46)=1,38, \mathrm{p}=0.25, \mathrm{r}=0.17)$, indicating that patients were not impaired at the 30-minute delayed recall.

Second, we tested performance on the one-week delayed recall test. In this case, significant group differences were found when testing recall after a week $(U=147, p$ $=0.008, \mathrm{r}=0.39)$. More importantly, we found a significant $\operatorname{group}(2) * \operatorname{delay}(2)$ interaction $(\mathrm{F}(2,46)=3.835, \mathrm{p}=0.029, \mathrm{r}=0.28)$. Results are presented in Figure 2. In the following paragraphs, we will describe the pattern of impairment for the left and right TLE patients separately.

We also tested performance accuracy on the recognition test (see Figure 2). Group differences were not found on the 30-minute delayed recognition task ( $U=202$, $p=0.18, r=-0.19)$ nor on the one-week delayed recognition task $(U=178.00, p=0.067$, $\mathrm{r}=-0.27)$. However, there was a significant interaction: $(\mathrm{F}(2,46)=4.788, \mathrm{p}=0.013, \mathrm{r}=$ $0.31)$.

\section{Delayed recall}

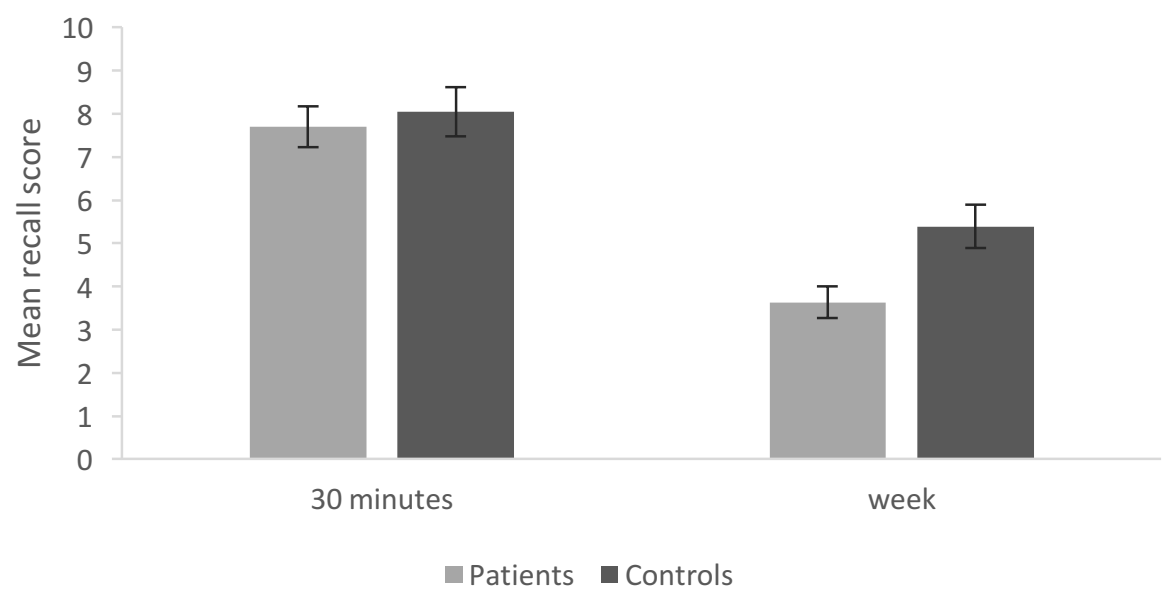




\section{Delayed recognition}



Figure 2. Verbal recall and recognition performance for patients with MTL resection and controls after 30 minutes and one week. Error bars represent SEM

\subsection{ALF in left TLE patients}

First, we tested performance decline on the 30-minute delayed recall. The MannWhitney test showed that verbal recall was not affected in left TLE patients, compared to controls, after 30 minutes $(U=99.50, p=0.48, r=-0.13)$. In addition, the group(2)* delay(2) interaction between immediate and 30-minute recall was not significant $(F(1,29)=1.06, p=0.31, r=0.19)$, indicating that patients were not impaired on the 30minute delayed recall. However, significant group differences were found when testing recall after a week $(U=51.00, p=0.008, r=-0.48)$. Furthermore, we found a significant group $(2) *$ delay $(2)$ interaction $(\mathrm{F}(1,29)=4.33, \mathrm{p}=0.047, \mathrm{r}=0,36)$, indicating ALF. Results are presented in Figure 3.

In addition, we tested the percentage of correct responses on the verbal recognition tasks. It was not affected in left TLE patients after 30 minutes $(U=92.20, p$ $=0.30, r=-0.19)$ and reached significance when tested after a one-week delay $(\mathrm{U}=$ $70.00, p=0.057, r=-0.34)$. In addition, there was a significant delay $(2) * \operatorname{group}(2)$ interaction when comparing the left TLE and control groups $(F(1,29)=9.220, p=$ $0.005, \mathrm{r}=0.49)$. 


\subsection{ALF in right TLE patients}

Verbal recall was not affected in right TLE patients, compared to controls, after 30 minutes $(\mathrm{U}=148.5 \mathrm{p}=0.79, \mathrm{r}=-0.04)$. In addition, the $\operatorname{group}(2) * \operatorname{delay}(2)$ interaction between immediate and 30-minute recall was not significant $(F(1,32)=1.01, p=0.32, r$ $=0.17$ ), indicating that patients were not impaired on the 30 -minute delayed recall.

However, verbal recall reached significance after a week $(U=96.00, p=0.057, r$ $=-0.46)$. In addition, there was a significant group $(2) *$ delay $(2)$ interaction $(F(1,32)=$ $6.59, \mathrm{p}=0.015, \mathrm{r}=0.41)$, suggesting that these patients have an accelerated rate of forgetting.

Furthermore, we tested the percentage of correct responses on the verbal recognition task. It was not affected in right TLE patients after 30 minutes ( $U=148.50$, $\mathrm{p}=0.88, \mathrm{r}=-0.03)$ nor after a one-week delay $(\mathrm{U}=108,00, \mathrm{p}=0.21, \mathrm{r}=-0.21)$. In addition, the group $(2) * \operatorname{delay}(2)$ interaction was not significant $(F(1,32)=0.987, p=$ $0.328, \mathrm{r}=0.17)$.

\section{Delayed Recall}

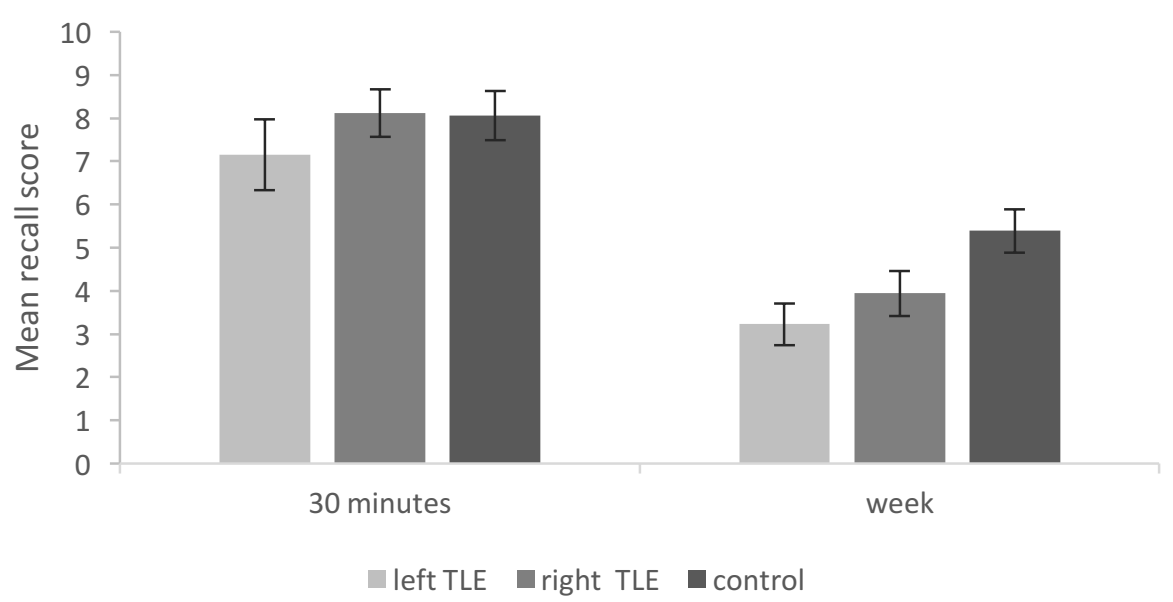




\section{Delayed Recognition}

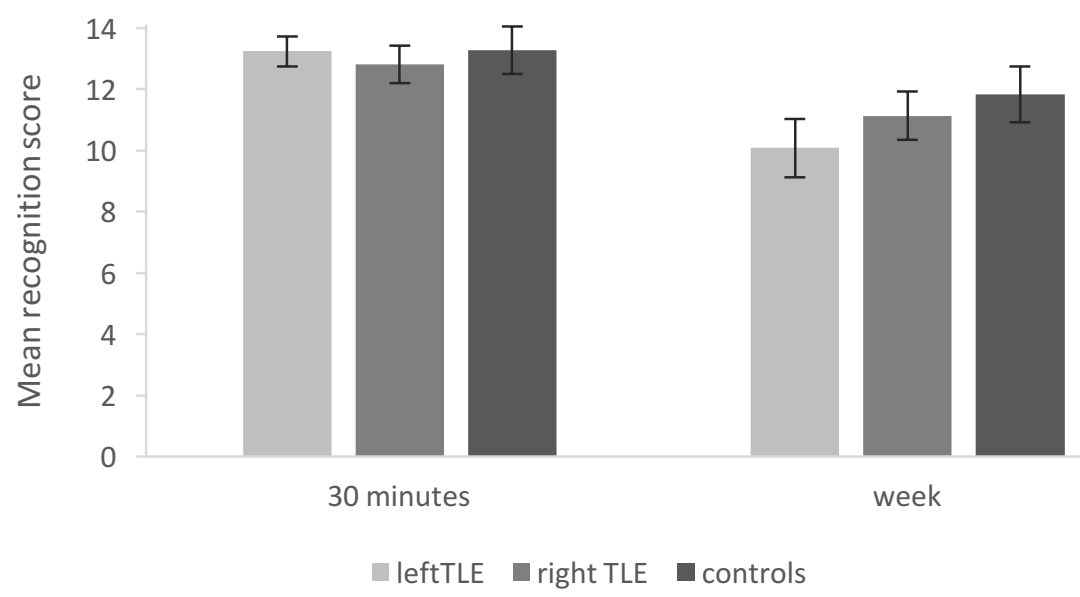

Figure 3. Verbal recall and recognition performance of patients with left and right MTL resection after 30 minutes and one week. Error bars represent SEM

\subsection{Comparing patients with and without overt seizures}

\begin{tabular}{|c|c|c|c|}
\hline & $\begin{array}{l}\text { Patients with } \\
\text { seizures }\end{array}$ & $\begin{array}{l}\text { Patients without } \\
\text { seizures }\end{array}$ & Matched Controls \\
\hline Learning trial 5 & $9.86(2.38)$ & $10.63(1.93)$ & $11.28(1.81)$ \\
\hline 30 min recall & $6.71(2.13)$ & $8.56(2.66)$ & $8.06(2.41)$ \\
\hline Week recall & $2.86(2.44)$ & $4.5(1.75)$ & $5.39(2.12)$ \\
\hline \multicolumn{4}{|c|}{$\begin{array}{l}\text { Table 4. Means and standard deviations of patients with }(\mathrm{N}=14) \text { and without }(\mathrm{N} \\
=16) \text { seizures, as well as the matched control group, on immediate, } 30 \text {-minute, } \\
\text { and one-week delayed recall. }\end{array}$} \\
\hline
\end{tabular}

We examined ALF in patients with $(\mathrm{N}=14)$ and without seizures $(\mathrm{N}=16)$. Of the patients with seizures, 6 were left TLE, and 8 were right TLE patients. Of the patients without seizures, 7 were left TLE, and 9 were right TLE patients. First, we tested for group differences on the last immediate recall trial, which approached significance for patients with seizures $(\mathrm{N}=14)$ and was not significant for patients without seizures $(\mathrm{N}=$ $16 ; \mathrm{U}=80.5, \mathrm{p}=0.08, \mathrm{r}=-0.31$ and $\mathrm{U}=112.5, \mathrm{p}=0.28, \mathrm{r}=-.19$, respectively).

Furthermore, verbal recall was not affected after 30 minutes in patients with and without seizures $(U=89, p=0.16, r=0.25$ and $U=120, p=0.40, r=0.14$, respectively). In addition, the group $(2) * \operatorname{delay}(2)$ interaction between immediate and 30-minute recall reached significance for patients with seizures and was non-significant for patients without seizures $(\mathrm{F}(1,33)=3.27, \mathrm{p}=0.08, \mathrm{r}=0.30$ and $\mathrm{F}(1,29)=1.08, \mathrm{p}=0.31, \mathrm{r}=0.18$, 
respectively). This result indicates that patients were not impaired at immediate and 30minute delayed recall, although patients with seizures showed a tendency toward episodic memory impairments in these early stages.

After one week, recall was affected in patients with seizures $(U=40.50, p=$ $0.001, r=-0.58)$, but not in patients without seizures $(U=106.50, p=0.19, r=-0.22)$. However, the group*delay interaction was significant for the seizure-free group $(F(1,34)=4.33, p=0.05, r=0.34)$, but not for the patients with seizures $(F(1,32)=2,36, p$ $=0.135, \mathrm{r}=0.26$ ). This finding indicates that it is actually the seizure-free group that shows an acceleration in forgetting.

Finally, we compared the performance of the two patient groups directly, finding no significant differences on the fifth learning trial $(U=92.5, p=0.41, r=-0.15)$. However, performance differed on the 30-minute and one-week delayed recall sessions $(\mathrm{U}=63.00, \mathrm{p}=0.04, \mathrm{r}=-0.37$ and $\mathrm{U}=56.5, \mathrm{p}=0.018, \mathrm{r}=-0.43$, respectively). The group * delay interaction was not significantly different between the two patient groups $(\mathrm{F}(1,28)=0.073, \mathrm{p}=0.79, \mathrm{r}=0.05)$.

\section{Discussion}

\subsection{ALF in resected TLE patients}

The current study investigated ALF in resected TLE patients. Patients with ALF typically perform normally on a 30-minute delayed recall test, but they show impaired performance after longer intervals (Butler \& Zeman, 2008). Furthermore, to demonstrate an actual acceleration in the rate of forgetting, it is important to find a delay*group interaction (Wilkinson et al., 2012). The pattern of impairment in the resected TLE patients in the current study aligned with ALF. That is, after matching the groups on learning, they showed a normal rate of forgetting on the 30-minute delayed recall test, but impaired performance after a week.

Although previous research has concentrated on TEA and non-resected TLE patients, the current study shows that ALF is also present in resected TLE patients. It is important to investigate ALF in patients with different clinical profiles because both hippocampal pathology and epileptiform activity influence memory performance (see Introduction). For example, in the study by Wilkinson et al. (2012), the volume of the 
remaining hippocampus correlated with performance, introducing possible confounding variations. As the patients in the current study had hippocampal resection, this influencing factor is removed.

The current results advocate for the extension of standard memory batteries for resected TLE patients. Furthermore, it urges clinicians to test ALF before and after surgery to investigate the extent to which surgery causes increases in ALF.

\subsection{Learning effects}

Both left and right TLE patients showed a deficit in the acquisition of new memories for verbal information. This might be partly due to the selection of our patients. Surgery was restricted to pre-operative patients with hippocampal sclerosis, as they are more resistant to post-surgery memory decline. However, these patients are also more likely to cope with memory problems before (and, therefore, also after) surgery. In agreement with this, Wilkinson et al. (2012) also found impairments during the learning phase in TLE patients with hippocampal pathology. Moreover, in their study, left hippocampal volume was associated with these memory deficits.

Furthermore, patient and control groups should be matched on initial learning to avoid scaling problems that can minimise the effects of ALF (Butler \& Zeman, 2008; Elliott et al., 2014; Isaac \& Mayes, 1999b; Wilkinson et al., 2012). In the current study, we decided to match the groups by excluding control participants who performed near ceiling level on immediate recall. An additional advantage was that we were able to use the standardized RAVLT without any adaptations (except the addition of a 7-day recall phase). Using this format will make it easier for clinicians to implement ALF testing in their standard memory batteries without increasing the examination time.

\subsection{Material-specific lateralisation of $A L F$}

The current study used a verbal word list as material. Previous studies have indicated a material-specific deficit in episodic memory for verbal material in left, but not right, TLE patients (Baxendale et al., 1998; Delaney et al., 1980; Hermann et al., 1997; Mungas et al., 1985). However, in the current literature, it is unclear whether ALF also shows this lateralisation effect. Whereas Blake et al. (2000) found ALF for verbal tests 
in left, but not right, non-resected TLE patients, Wilkinson et al. (2012) found that both left and right TLE patients had ALF for verbal material. The results of the current study align with the results of Wilkinson et al. (2012): both left and right TLE patients showed ALF, demonstrated by a delay*group interaction.

The differences in verbal lateralisation effects between the Blake et al.(2000) study, the Wilkinson et al. (2012) study, and the current study might be caused by their focus on different patient profiles. Blake et al. (2000) categorised TLE patients based on left or right seizure activity, with or without hippocampal damage. In contrast, Wilkinson et al. (2012) only included patients with demonstrable hippocampal damage. As the current study was restricted to resected TLE patients, considerable hippocampal damage was guaranteed. This seems to indicate that the presence or absence of hippocampal damage in the right MTL is a defining factor in verbal ALF effects in right TLE patients.

However, we need to take into account that the type of verbal material (word list vs. story) might influence the results. Elliot et al. (2014) pointed out that the lack of standardized methods to investigate ALF is likely to explain the mixed findings on ALF in TLE patients. In the absence of standardized tests, researchers either create their own material or adapt existing standard tests. Therefore, more studies using different types of material are required to speculate any further on this issue.

Overall, the current results suggest that lateralisation effects on ALF in the episodic system are minimal in resected TLE patients. It seems that both left and right MTL damage causes ALF for verbal information, agreeing with Glikmann-Johnston et al. (2008) that there is a bilateral dynamic interaction between the MTL structures during episodic memory events. In other words, the episodic memory system in the MTL is bilateral, although it might be skewed to the left on verbal tasks. A similar system has been proposed for semantic memory in the lateral temporal regions (Lambon Ralph, Ehsan, Baker, \& Rogers, 2012; Rice, Hoffman, \& Lambon Ralph, 2015). This skewedness allows an intact left MTL to function relatively well with a mildly damaged right MTL, but the reverse pattern causes clear verbal episodic memory problems. This means that left MTL damage is more frequently associated with verbal memory problems and ALF. However, severe right MTL damage (such as en bloc resection) will also cause ALF of verbal information. 


\subsection{ALF for recognition memory}

In the current study, neither left nor right TLE patients had impaired delayed recognition after 30 minutes nor after a week. However, for the left TLE patients, there was a delay*group interaction, indicating an acceleration in forgetting compared to controls. This result agrees with a previous study that showed ALF on recognition memory in TEA patients (although they did show a significant group difference after a week; Hoefeijzers, Dewar, Della Sala, Zeman, \& Butler, 2013).

Both recall and recognition depend on recollective (episodic) memory, which supports the ability to remember the exact event in which it occurred. However, recognition memory can also depend on familiarity memory, which refers to the ability to know that the item was presented without remembering the episode itself (Isaac \& Mayes, 1999a; Manns, Hopkins, \& Squire, 2003). Recollective (episodic) memory has been shown to depend mainly on the hippocampus, whereas familiarity additionally depends on surrounding lateral temporal regions (Wolk \& Dickerson, 2011). Studies on ALF have suggested including both measures (Elliott et al., 2014).

\subsection{Comparing patients with and without seizures}

Overt seizures have been associated with ALF (Mameniskiene et al., 2006; O'Connor et al., 1997; Wilkinson et al., 2012). Therefore, we investigated the memory decline pattern separately in patients with and without overt seizures. The results indicated that the patient group without seizures shows an acceleration in forgetting, which demonstrates that the presence of ALF in the current patient sample cannot be attributed to overt seizures. Consequently, MTL damage is the most obvious reason for ALF in the current patient group. However, we cannot discard the presence of subclinical epileptiform activity that can mediate the ALF effect. Future research should test this possibility. The results of the present study seem to indicate that the memory problems in the seizure group might arise at an earlier stage and are not specific to long-term memory impairments after $24 \mathrm{~h}$. Patients with seizures performed worse than patients without seizures on the 30-minute delayed recall. In addition, performance differences 
on immediate and 30-minute recall reached significance when compared to the control group $(\mathrm{p}=0.08)$, supporting the possibility of an earlier memory problem. Likewise, seizure frequency has been associated with episodic memory impairments in the immediate and 30-minute recall stages (Voltzenlogel, Vignal, Hirsch, \& Manning, 2014). However, future research with more patients is required to study this further.

\subsection{Limitations}

The current study clearly demonstrates ALF in resected TLE patients. This shows that standard memory batteries do not capture the full range of memory problems in TLE patients and advocates for the inclusion of ALF tests. However, it is important to find the origin of ALF. Resected TLE patients have suffered from long-standing preoperative neural changes caused by the epileptic seizures themselves. In our study, surgery was restricted to pre-operative patients with hippocampal sclerosis, as they are more resistant to post-surgery memory decline. However, these patients are also more likely to struggle with memory problems before (and, therefore, also after) surgery, making this a confounding factor. Important insight can be gained by comparing preand post-operative results. This type of longitudinal study can reveal the extent of the damage to the episodic memory system caused by the operation that cannot be attributed to pre-operative neural changes. Finally, standard en bloc resection includes hippocampal removal, but it also affects surrounding lateral regions, which might have contributed to the memory decline in the resected TLE patients.

\section{References}

Barr, W. B., Goldberg, E., Wasserstein, J., \& Novelly, R. A. (1990). Retrograde amnesia following unilateral temporal lobectomy. Neuropsychologia, 28(3), 243255. http://doi.org/10.1016/0028-3932(90)90018-J

Baxendale, S. A., van Paesschen, W., Thompson, P. J., Connelly, A., Duncan, J. S., Harkness, W. F., \& Shorvon, S. D. (1998). The relationship between quantitative MRI and neuropsychological functioning in temporal lobe epilepsy. Epilepsia, 39(2), 158-66. Retrieved from http://www.ncbi.nlm.nih.gov/pubmed/9577995

Bell, B. D. (2006). WMS-III Logical Memory performance after a two-week delay in 
temporal lobe epilepsy and control groups. Journal of Clinical and Experimental Neuropsychology, 28(8), 1435-43. http://doi.org/10.1080/13803390500434367

Bell, B. D., Fine, J., Dow, C., Seidenberg, M., \& Hermann, B. P. (2005). Temporal lobe epilepsy and the selective reminding test: the conventional 30-minute delay suffices. Psychological Assessment, 17(1), 103-9. http://doi.org/10.1037/10403590.17 .1 .103

Bell, B., Lin, J. J., Seidenberg, M., \& Hermann, B. (2011). The neurobiology of cognitive disorders in temporal lobe epilepsy. Nature Reviews. Neurology, 7(3), 154-64. http://doi.org/10.1038/nrneurol.2011.3

Blake, R. V, Wroe, S. J., Breen, E. K., \& McCarthy, R. A. (2000). Accelerated forgetting in patients with epilepsy: evidence for an impairment in memory consolidation. Brain : A Journal of Neurology, 123 Pt 3, 472-83. Retrieved from http://www.ncbi.nlm.nih.gov/pubmed/10686171

Butler, C. R., Bhaduri, A., Acosta-Cabronero, J., Nestor, P. J., Kapur, N., Graham, K. S., ... Zeman, A. Z. (2009). Transient epileptic amnesia: regional brain atrophy and its relationship to memory deficits. Brain, 132(2), 357-368. http://doi.org/10.1093/brain/awn336

Butler, C. R., Graham, K. S., Hodges, J. R., Kapur, N., Wardlaw, J. M., \& Zeman, A. Z. J. (2007). The syndrome of transient epileptic amnesia. Annals of Neurology, 61(6), 587-98. http://doi.org/10.1002/ana.21111

Butler, C. R., \& Zeman, A. (2008). A case of transient epileptic amnesia with radiological localization. Nature Clinical Practice Neurology, 4(9), 516-521. http://doi.org/10.1038/ncpneuro0857

Butler, C. R., \& Zeman, A. Z. (2008). Recent insights into the impairment of memory in epilepsy: transient epileptic amnesia, accelerated long-term forgetting and remote memory impairment. Brain : A Journal of Neurology, 131(Pt 9), 2243-63. http://doi.org/10.1093/brain/awn127

Cabeza, R., \& Nyberg, L. (2000). Imaging Cognition II: An Empirical Review of 275 PET and fMRI Studies. Journal of Cognitive Neuroscience, 12(1), 1-47. http://doi.org/10.1162/08989290051137585 
Cassel, A., Morris, R., Koutroumanidis, M., \& Kopelman, M. (2016). Forgetting in temporal lobe epilepsy: When does it become accelerated? Cortex, 78, 70-84. http://doi.org/10.1016/j.cortex.2016.02.005

Delaney, R. C., Rosen, A. J., Mattson, R. H., \& Novelly, R. A. (1980). Memory function in focal epilepsy: a comparison of non-surgical, unilateral temporal lobe and frontal lobe samples. Cortex; a Journal Devoted to the Study of the Nervous System and Behavior, 16(1), 103-17. Retrieved from http://www.ncbi.nlm.nih.gov/pubmed/6769639

Elliott, G., Isaac, C. L., \& Muhlert, N. (2014). Measuring forgetting: a critical review of accelerated long-term forgetting studies. Cortex; a Journal Devoted to the Study of the Nervous System and Behavior, 54(100), 16-32. http://doi.org/10.1016/j.cortex.2014.02.001

Giovagnoli, A. R., Casazza, M., \& Avanzini, G. (1995). Visual Learning on a Selective Reminding Procedure and Delayed Recall in Patients with Temporal Lobe Epilepsy. Epilepsia, 36(7), 704-711. http://doi.org/10.1111/j.15281157.1995.tb01050.x

Gleissner, U., Helmstaedter, C., \& Elger, C. E. (1998). Right hippocampal contribution to visual memory: a presurgical and postsurgical study in patients with temporal lobe epilepsy. Journal of Neurology, Neurosurgery, and Psychiatry, 65(5), 665-9. Retrieved from http://www.ncbi.nlm.nih.gov/pubmed/9810934

Glikmann-Johnston, Y., Saling, M. M., Chen, J., Cooper, K. A., Beare, R. J., \& Reutens, D. C. (2008). Structural and functional correlates of unilateral mesial temporal lobe spatial memory impairment. Brain, 131(11), 3006-3018. http://doi.org/10.1093/brain/awn213

Helmstaedter, C., Hauff, M., \& Elger, C. E. (1998). Ecological Validity of ListLearning Tests and Self-Reported Memory in Healthy Individuals and Those with Temporal Lobe Epilepsy. Journal of Clinical and Experimental Neuropsychology (Neuropsychology, Development and Cognition: Section A), 20(3), 365-375. http://doi.org/10.1076/jcen.20.3.365.824

Hermann, B. P., Seidenberg, M., Schoenfeld, J., \& Davies, K. (1997). Neuropsychological characteristics of the syndrome of mesial temporal lobe 
epilepsy. Archives of Neurology, 54(4), 369-76. Retrieved from http://www.ncbi.nlm.nih.gov/pubmed/9109737

Hoefeijzers, S., Dewar, M., Della Sala, S., Butler, C., \& Zeman, A. (2015). Accelerated long-term forgetting can become apparent within 3-8 hours of wakefulness in patients with transient epileptic amnesia. Neuropsychology, 29(1), 117-25. http://doi.org/10.1037/neu0000114

Hoefeijzers, S., Dewar, M., Della Sala, S., Zeman, A., \& Butler, C. (2013). Accelerated long-term forgetting in transient epileptic amnesia: An acquisition or consolidation deficit? Neuropsychologia, 51(8), 1549-1555. http://doi.org/10.1016/j.neuropsychologia.2013.04.017

Isaac, C. L., \& Mayes, A. R. (1999a). Rate of forgetting in amnesia: I. Recall and recognition of prose. Journal of Experimental Psychology. Learning, Memory, and Cognition, 25(4), 942-62. Retrieved from http://www.ncbi.nlm.nih.gov/pubmed/10439502

Isaac, C. L., \& Mayes, A. R. (1999b). Rate of forgetting in amnesia: II. Recall and recognition of word lists at different levels of organization. Journal of Experimental Psychology. Learning, Memory, and Cognition, 25(4), 963-77. Retrieved from http://www.ncbi.nlm.nih.gov/pubmed/10439503

Jansari, A. S., Davis, K., McGibbon, T., Firminger, S., \& Kapur, N. (2010). When ?long-term memory? no longer means ?forever?: Analysis of accelerated long-term forgetting in a patient with temporal lobe epilepsy. Neuropsychologia, 48(6), 1707-1715. http://doi.org/10.1016/j.neuropsychologia.2010.02.018

Kapur, N. (1990). Transient epileptic amnesia: A clinically distinct form of neurological memory disorder. In H. J. Markowitsch (Ed.), Transient global amnesia and related disorders (pp. 140-151). New York: Hogrefe and Huber.

Kapur, N., Millar, J., Colbourn, C., Abbott, P., Kennedy, P., \& Docherty, T. (1997). Very Long-Term Amnesia in Association with Temporal Lobe Epilepsy: Evidence for Multiple-Stage Consolidation Processes. Brain and Cognition, 35(1), 58-70. http://doi.org/10.1006/brcg.1997.0927

Kaufman, A. S., \& Lichtenberger, E. O. (2006). Assessing Adolescent and Adult Intelligence. Hoboken: Wiley. 
Kemp, S., Illman, N. A., Moulin, C. J. A., \& Baddeley, A. D. (2012). Accelerated longterm forgetting (ALF) and transient epileptic amnesia (TEA): Two cases of epilepsy?related memory disorder. Epilepsy \& Behavior, 24(3), 382-388. http://doi.org/10.1016/j.yebeh.2012.04.119

Kilpatrick, C., Murrie, V., Cook, M., Andrewes, D., Desmond, P., \& Hopper, J. (1997). Degree of left hippocampal atrophy correlates with severity of neuropsychological deficits. Seizure, 6(3), 213-8. Retrieved from http://www.ncbi.nlm.nih.gov/pubmed/9203250

Lambon Ralph, M. A., Ehsan, S., Baker, G. A., \& Rogers, T. T. (2012). Semantic memory is impaired in patients with unilateral anterior temporal lobe resection for temporal lobe epilepsy. Brain, 135(Pt 1), 242-58. http://doi.org/10.1093/brain/awr325

Lee, T. M. C., Yip, J. T. H., \& Jones-Gotman, M. (2002). Memory deficits after resection from left or right anterior temporal lobe in humans: a meta-analytic review. Epilepsia, 43(3), 283-91. Retrieved from http://www.ncbi.nlm.nih.gov/pubmed/11906514

Lencz, T., McCarthy, G., Bronen, R. A., Scott, T. M., Inserni, J. A., Sass, K. J., ... Spencer, D. D. (1992). Quantitative magnetic resonance imaging in temporal lobe epilepsy: Relationship to neuropathology and neuropsychological function. Annals of Neurology, 31(6), 629-637. http://doi.org/10.1002/ana.410310610

Leys, C., \& Schumann, S. (2010). A nonparametric method to analyze interactions: The adjusted rank transform test. Journal of Experimental Social Psychology, 46(4), 684-688. http://doi.org/10.1016/j.jesp.2010.02.007

Lucchelli, F., \& Spinnler, H. (1998). Ephemeral New Traces and Evaporated Remote Engrams: A Form of Neocortical Temporal Lobe Amnesia? A Preliminary Case Report. Neurocase, 4(6), 447-459. http://doi.org/10.1080/13554799808410638

Mameniskiene, R., Jatuzis, D., Kaubrys, G., \& Budrys, V. (2006). The decay of memory between delayed and long-term recall in patients with temporal lobe epilepsy. Epilepsy and Behavior, 8(1), 278-288. http://doi.org/10.1016/j.yebeh.2005.11.003

Manns, J. R., Hopkins, R. O., \& Squire, L. R. (2003). Semantic memory and the human 
hippocampus. Neuron, 38(1), 127-33. Retrieved from

http://www.ncbi.nlm.nih.gov/pubmed/12691670

Martin, R. C., Loring, D. W., Meador, K. J., Lee, G. P., Thrash, N., \& Arena, J. G. (1991). Impaired long-term retention despite normal verbal learning in patients with temporal lobe dysfunction. Neuropsychology, 5(1), 3-12. http://doi.org/10.1037/0894-4105.5.1.3

Mayes, A., Isaac, C., Holdstock, J., Cariga, P., Gummer, A., \& Roberts, N. (2003). Long-Term Amnesia: A Review and Detailed Illustrative Case Study. Cortex, 39(4-5), 567-603. http://doi.org/10.1016/S0010-9452(08)70855-4

McGibbon, T., \& Jansari, A. S. (2013). Detecting the onset of accelerated long-term forgetting: Evidence from temporal lobe epilepsy. Neuropsychologia, 51(1), 114122. http://doi.org/10.1016/j.neuropsychologia.2012.11.004

Meehl, P. E. (1970). Nuisance variables and the ex post facto design. In M. Radner \& S. Winokur (Eds.), Analyses of Theories and Methods of Physics and Psychology (pp. 373-402). Minneapolis: University of Minnesota Press.

Miller, L. A., Flanagan, E., Mothakunnel, A., Mohamed, A., \& Thayer, Z. (2015). Old dogs with new tricks: Detecting accelerated long-term forgetting by extending traditional measures. Epilepsy \& Behavior, 45, 205-211. http://doi.org/10.1016/j.yebeh.2015.01.024

Milner, B. (1971). Interhemispheric differences in the localization of psychological processes in man. British Medical Bulletin, 27(3), 272-7. Retrieved from http://www.ncbi.nlm.nih.gov/pubmed/4937273

Moscovitch, M., Cabeza, R., Winocur, G., \& Nadel, L. (2016). Episodic Memory and Beyond: The Hippocampus and Neocortex in Transformation. Annual Review of Psychology, 67, 105-34. http://doi.org/10.1146/annurev-psych-113011-143733

Muhlert, N., Milton, F., Butler, C. R., Kapur, N., \& Zeman, A. Z. (2010). Accelerated forgetting of real-life events in Transient Epileptic Amnesia. Neuropsychologia, 48(11), 3235-3244. http://doi.org/10.1016/j.neuropsychologia.2010.07.001

Mungas, D., Ehlers, C., Walton, N., \& McCutchen, C. B. (1985). Verbal learning differences in epileptic patients with left and right temporal lobe foci. Epilepsia, 
26(4), 340-5. Retrieved from http://www.ncbi.nlm.nih.gov/pubmed/4006893

Nadel, L., \& Moscovitch, M. (1997). Memory consolidation, retrograde amnesia and the hippocampal complex. Current Opinion in Neurobiology, 7(2), 217-27. Retrieved from http://www.ncbi.nlm.nih.gov/pubmed/9142752

O’Connor, M., Sieggreen, M. A., Ahern, G., Schomer, D., \& Mesulam, M. (1997). Accelerated Forgetting in Association with Temporal Lobe Epilepsy and Paraneoplastic Encephalitis. Brain and Cognition, 35(1), 71-84. http://doi.org/10.1006/brcg.1997.0928

Pauli, E., Hildebrandt, M., Romstock, J., Stefan, H., \& Blumcke, I. (2006). Deficient memory acquisition in temporal lobe epilepsy is predicted by hippocampal granule cell loss. Neurology, 67(8), 1383-1389. http://doi.org/10.1212/01.wnl.0000239828.36651.73

Piazzini, A., Canevini, M. P., Maggiori, G., \& Canger, R. (2001). The perception of memory failures in patients with epilepsy. European Journal of Neurology, 8(6), 613-20. Retrieved from http://www.ncbi.nlm.nih.gov/pubmed/11784346

Rausch, R., \& Babb, T. L. (1993). Hippocampal neuron loss and memory scores before and after temporal lobe surgery for epilepsy. Archives of Neurology, 50(8), 812-7. Retrieved from http://www.ncbi.nlm.nih.gov/pubmed/8352666

Reminger, S. L., Kaszniak, A. W., Labiner, D. M., Littrell, L. D., David, B. T., Ryan, L., ... Kaemingk, K. L. (2004). Bilateral hippocampal volume predicts verbal memory function in temporal lobe epilepsy. Epilepsy \& Behavior, 5(5), 687-695. http://doi.org/10.1016/j.yebeh.2004.06.006

Rey, A. (1958). L'Examen Clinique en Psychologie (Press Univ). Paris.

Ricci, M., Mohamed, A., Savage, G., \& Miller, L. A. (2015). Disruption of learning and long-term retention of prose passages in patients with focal epilepsy. Epilepsy \& Behavior, 51, 104-111. http://doi.org/10.1016/j.yebeh.2015.06.016

Rice, G. E., Hoffman, P., \& Lambon Ralph, M. A. (2015). Graded specialization within and between the anterior temporal lobes. Annals of the New York Academy of Sciences, 1359(1), 84-97. http://doi.org/10.1111/nyas.12951

Squire, L. R., \& Alvarez, P. (1995). Retrograde amnesia and memory consolidation: a 
neurobiological perspective. Current Opinion in Neurobiology, 5(2), 169-77.

Retrieved from http://www.ncbi.nlm.nih.gov/pubmed/7620304

Tramoni, E., Felician, O., Barbeau, E. J., Guedj, E., Guye, M., Bartolomei, F., \&

Ceccaldi, M. (2011). Long-term consolidation of declarative memory: insight from temporal lobe epilepsy. Brain, 134(3), 816-831.

http://doi.org/10.1093/brain/awr002

Voltzenlogel, V., Vignal, J.-P., Hirsch, E., \& Manning, L. (2014). The influence of seizure frequency on anterograde and remote memory in mesial temporal lobe epilepsy. Seizure, 23(9), 792-8. http://doi.org/10.1016/j.seizure.2014.06.013

Wilkinson, H., Holdstock, J. S., Baker, G., Herbert, A., Clague, F., \& Downes, J. J. (2012). Long-term accelerated forgetting of verbal and non-verbal information in temporal lobe epilepsy. Cortex, 48(3), 317-332. http://doi.org/10.1016/j.cortex.2011.01.002

Wolk, D. A., \& Dickerson, B. C. (2011). Fractionating verbal episodic memory in Alzheimer's disease. NeuroImage, 54(2), 1530-9. http://doi.org/10.1016/j.neuroimage.2010.09.005

Zeman, A., \& Butler, C. (2010). Transient epileptic amnesia. Current Opinion in Neurology, 23(6), 610-616. http://doi.org/10.1097/WCO.0b013e32834027db

Zeman, A., Butler, C., Muhlert, N., \& Milton, F. (2013). Novel forms of forgetting in temporal lobe epilepsy. Epilepsy \& Behavior, 26, 335-342.

http://doi.org/10.1016/j.yebeh.2012.09.030 